INSTITUT NATIONAL DE RECHERCHE EN INFORMATIQUE ET EN AUTOMATIQUE

\title{
Approximate Transmission Conditions through a rough thin layer. The case of the periodic roughness
}

\author{
Ionel Ciuperca — Mohammed Jai — Clair Poignard
}

$\mathbf{N}^{\circ} 6812$

Janvier 2009

Thème NUM 



\title{
Approximate Transmission Conditions through a rough thin layer. The case of the periodic roughness
}

\author{
Ionel Ciuperca *, Mohammed Ja用, Clair Poignard田 \\ Thème NUM — Systèmes numériques \\ Équipes-Projets MC2 \\ Rapport de recherche $\mathrm{n}^{\circ} 6812$ - Janvier 2009 - 23 pages
}

\begin{abstract}
We study the behavior of the steady-state voltage potentials in a material composed by a bidimensional medium surrounded by a rough thin layer and embedded in an ambient medium. The roughness of the layer is supposed to be $\varepsilon$-periodic, $\varepsilon$ beeing the small thickness of the layer. We build approximate transmission conditions in order to replace the rough thin layer by these conditions on the boundary of the interior material. This paper extends previous works [8, 7] of the third author, in which the layer had constant or weakly oscillating thickness.
\end{abstract}

Key-words: Asymptotic analysis, Laplace equations

* Université de Lyon, Université Lyon 1, CNRS, UMR 5208, Institut Camille Jordan, Bat. Braconnier, 43 boulevard du 11 novembre 1918, F - 69622 Villeurbanne Cedex, France

† ICJ CNRS-UMR 5208, INSA de Lyon, Bât. Léonard de Vinci, 20 avenue A. Einstein 69621 Villeurbanne cedex, France

¥ INRIA Bordeaux-Sud-Ouest, Institut de Mathématiques de Bordeaux, CNRS UMR 5251 \& Université de Bordeaux1, 351 cours de la Libération, 33405 Talence Cedex, France

Centre de recherche INRIA Bordeaux - Sud Ouest Domaine Universitaire - 351, cours de la Libération 33405 Talence Cedex 
Conditions de transmission approchées pour une couche mince rugueuse. Cas d'une rugosité périodique

Résumé :

Mots-clés : Analyse Asymptotique, Equations de Laplace 


\section{Contents}

1 Introduction 4

1.1 Motivations . . . . . . . . . . . . . . . . . . 4

1.2 Statement of the problem . . . . . . . . . . . . . . 5

1.3 Main result . . . . . . . . . . . . . . . . . . . . . . 6

2 Preliminary analysis 8

2.1 The equivalent problem in a tubular neighborhood of $\Gamma$. . . . 8

2.2 The problem in local coordinates . . . . . . . . . . . . . 10

2.3 Preliminary result . . . . . . . . . . . . . . . . . . . . . . . 12

3 Formal asymptotics 15

3.1 Zeroth order approximation . . . . . . . . . . . . . . . 15

$3.2 \quad$ First order approximation . . . . . . . . . . . . . . . . . . . 19

\begin{tabular}{|lll}
4 & Justification of the expansion & 21
\end{tabular} 


\section{Introduction}

This paper deals with the construction of approximate transmission conditions equivalent to a "rough" thin layer for the steady-state voltage potentials. The roughness of the layer is supposed to be $\varepsilon$-periodic, and the thickness of the layer is of order $\varepsilon, \varepsilon$ being a small positive parameter.

\subsection{Motivations}

Rough layers appear in many research area. For instance in geophysics the bottom of the oceans and the shores are rough with respect to the large scale flow, in tribology surfaces present a granular aspect at the microscopic scale. The motivation of this paper comes from a collaborative research with Schlumberger on the electric modelization of silty soils.

In the simplest modelizations silty soils are composed by water, clay and quartz. The clay leaves are organized on the surface of quartz grains. Because of their electrical properties, the presence or the lack of clay leaves changes considerably the effective properties of soils. Actually, since they are electrically charged clay leaves stick the water molecules around them. This changes the electric properties of the water so-called bound water or hygroscopic water [10. 9. This phenomenon occurs on few layers of water molecules, therefore the hygroscopic water is modelized by a rough thin layer. The assembly of quartz grain and clay leaves is modelized by an electrically homogeneous domain with non-zero conductivity, while the ambiant medium is free water (see Fig 1).

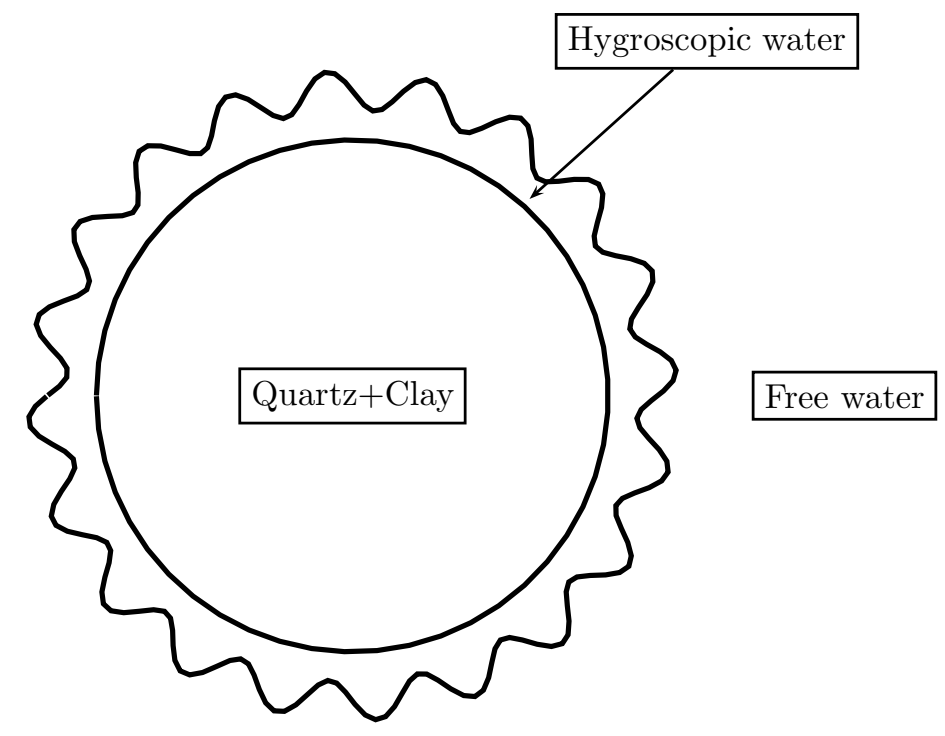

Figure 1: Scheme of the assembly of quartz grain and clay surrounded by a thin layer of hygroscopic water. The ambient medium is composed by free water. 
The goal of this paper is to understand the effect of the hygroscopic water on the electric steady-state potential.

\subsection{Statement of the problem}

For sake of simplicity, we deal with the bidimensional case, however the threedimensional case may be obtained in the same way up to few appropriate modifications. Let $\Omega$ be a smooth bounded domain of $\mathbb{R}^{2}$ with connected boundary $\partial \Omega$. For $\varepsilon>0$, we split $\Omega$ into three subdomains: $\mathcal{D}^{1}, \mathcal{D}_{\varepsilon}^{m}$ and $\mathcal{D}_{\varepsilon}^{0}$. $\mathcal{D}_{1}$ is a smooth domain strictly embedded in $\Omega$ (see Fig. 2).

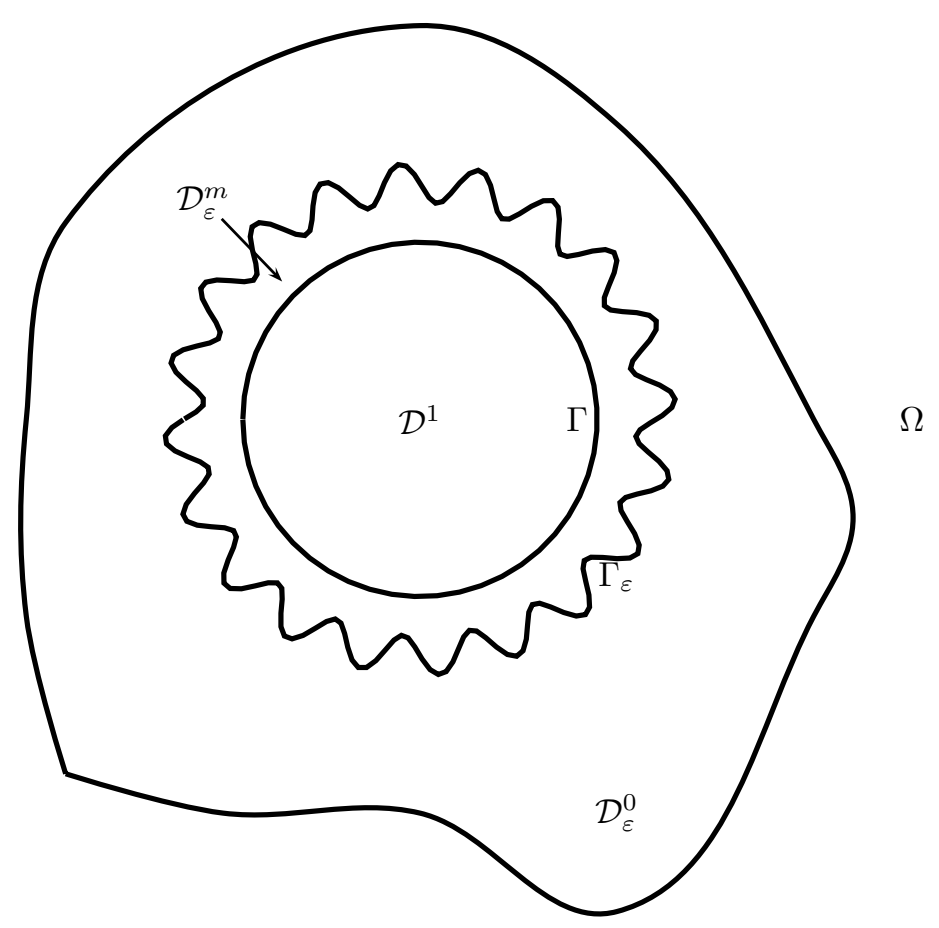

Figure 2: Geometry of the problem.

We denote by $\Gamma$ its connected boundary. The domain $\mathcal{D}_{\varepsilon}^{m}$ is a thin oscillating layer surrounding $\mathcal{D}_{1}$. We denote by $\Gamma_{\varepsilon}$ the oscillating boundary of $\mathcal{D}_{\varepsilon}^{m}$ :

$$
\Gamma_{\varepsilon}=\partial \mathcal{D}_{\varepsilon}^{m} \backslash \Gamma
$$

The domain $\mathcal{D}_{\varepsilon}^{0}$ is defined by:

$$
\mathcal{D}_{\varepsilon}^{0}=\Omega \backslash \overline{\left(\mathcal{D}^{1} \cup \mathcal{D}_{\varepsilon}^{m}\right)} .
$$

We also denote by

$$
\mathcal{D}^{0}=\Omega \backslash \overline{\mathcal{D}^{1}}
$$


We define the piecewise constant function $\sigma: \Omega \rightarrow \mathbb{R}$ by

$$
\sigma(z)=\left\{\begin{array}{l}
\sigma_{1}, \text { if } z \in \mathcal{D}^{1}, \\
\sigma_{m}, \text { if } z \in \mathcal{D}_{\varepsilon}^{m}, \\
\sigma_{0}, \text { if } z \in \mathcal{D}_{\varepsilon}^{0} .
\end{array}\right.
$$

where $\sigma_{1}, \sigma_{m}$ and $\sigma_{0}$ are given positive constant 1 .

Let $g$ belong to $H^{s}(\partial \Omega)$, for $s \geq 1 / 2$. We consider the unique solution $u^{\varepsilon}$ to the following problem:

$$
\begin{aligned}
& \nabla \cdot\left(\sigma \nabla u^{\varepsilon}\right)=0, \text { in } \Omega, \\
& \left.u^{\varepsilon}\right|_{\partial \Omega}=g .
\end{aligned}
$$

Observe that for all $\varepsilon>0$, the domains $\Omega, \mathcal{D}^{1}, \mathcal{D}_{\varepsilon}^{m}$ and $\mathcal{D}_{\varepsilon}^{0}$ are smooth. Hence the above function $u^{\varepsilon}$ belongs to $H^{1}(\Omega)$ and moreover it belongs to $H^{s+1 / 2}\left(\mathcal{D}^{1}\right)$, $H^{s+1 / 2}\left(\mathcal{D}_{\varepsilon}^{0}\right)$ and $H^{s+1 / 2}\left(\mathcal{D}_{\varepsilon}^{m}\right)$. Our aim is to give the first two terms of the asymptotic expansion of $u^{\varepsilon}$ for $\varepsilon$ tending to zero.

Several papers are devoted to rough boundaries and derivations of equivalent boundary conditions [1, 2, 3, 6. In a recent article Basson and Gérard-Varet 4] derive approximate boundary condition for a boundary with random roughness. The analysis of these previous papers is essentially based on the construction of the so-called "wall law", which is a boundary condition imposed on an artificial boundary inside the domain. The wall law only reflects the large scale effect on the oscillations. In this paper we are definitely interested in approximated transmission conditions, therefore we cannot apply straightforward the previous results to our problem. In a previous article [7, the third author derives the first order approximate boundary condition for a thin weakly oscillating layer. This paper is an extension of [7] to the case of an $\varepsilon$-periodic thin layer with thickness of order $\varepsilon$. The main idea of the analysis comes from the paper of Abboud et Ammari [1] and is completely different from the analysis of [7, which was based on an appropriate change of variables. Here the boundary layer corrector are obtained by solving an elliptic partial differential equation in an appropriate infinite band with width equal to 1 . Well-posedness of the problems and optimal error are given to justify our expansion.

The outline of the paper is the following: in Section 2 we perform a suitable change of variables in order to derive our asymptotics in the simplest way. We also give a preliminary result, which will be useful to prove the formal asymptotics of Section 3 . The Section 4 is devoted to the justification of our equivalent transmission condition. Present now our main result.

\subsection{Main result}

Let $\mathbb{T}$ be the torus $\mathbb{R} / \mathbb{Z}$. Since $\Gamma$ is a smooth closed curve of $\mathbb{R}^{2}$ of length 1 , it is parameterized by its curvilinear coordinate:

$$
\Gamma=\{\Psi(\theta), \theta \in \mathbb{T}\}
$$

Denote by $n$ the exterior normal to $\Gamma$. The rough boundary $\Gamma_{\varepsilon}$ is defined by

$$
\Gamma_{\varepsilon}=\left\{\Psi_{\varepsilon}(\theta)=\Psi(\theta)+\varepsilon f(\theta / \varepsilon) n(\theta), \theta \in \mathbb{T}\right\},
$$

\footnotetext{
${ }^{1}$ The same following results hold if $\sigma_{0}, \sigma_{1}$, and $\sigma_{m}$ are given complex numbers with imaginary parts (and respectively real parts) with the same sign.
} 
where $f$ is a smooth 1 -periodic and positive function. The closed curves $\mathcal{C}_{1}$ and $\mathcal{C}_{0}$ are defined by:

$$
\begin{aligned}
& \mathcal{C}_{0}=\{0\} \times \mathbb{T} \\
& \mathcal{C}_{1}=\{(f(y), y), \forall y \in \mathbb{T}\}
\end{aligned}
$$

$\mathcal{C}_{1}$ and $\mathcal{C}_{0}$ have the trigonometric orientation. The exterior normal to $\mathcal{C}_{1}$ is denoted by $n_{\mathcal{C}_{1}}$ and $n_{\mathcal{C}_{0}}$ is the exterior normal to $\mathcal{C}_{0}$ :

$$
n_{\mathcal{C}_{0}}=\left(\begin{array}{l}
1 \\
0
\end{array}\right), \quad n_{\mathcal{C}_{1}}=\frac{1}{\sqrt{1+\left(f^{\prime}(y)\right)^{2}}}\left(\begin{array}{c}
1 \\
-f^{\prime}(y)
\end{array}\right) .
$$

Define now the couple $\left(A^{0}, a^{0}\right)$, where $A^{0}$ is a continuous vector-field and $a^{0}$ a constant vector, which is the unique solution of the following problem:

$$
\begin{aligned}
& \Delta A^{0}=0, \text { in } \mathbb{R} \times \mathbb{T}, \\
& \left.\sigma_{0} \partial_{n} A^{0}\right|_{\mathcal{C}_{1}^{+}}-\left.\sigma_{m} \partial_{n} A^{0}\right|_{\mathcal{C}_{1}^{-}}=\left(\sigma_{m}-\sigma_{0}\right) n_{\mathcal{C}_{1}}, \\
& \left.\sigma_{m} \partial_{n} A^{0}\right|_{\mathcal{C}_{0}^{+}}-\left.\sigma_{1} \partial_{n} A^{0}\right|_{\mathcal{C}_{0}^{-}}=-\left(\sigma_{m}-\sigma_{0}\right) n_{\mathcal{C}_{0}}, \\
& A^{0} \rightarrow_{x \rightarrow-\infty} 0, A^{0} \rightarrow_{x \rightarrow+\infty} a^{0} .
\end{aligned}
$$

We denote by $D_{1}$ and $D_{2}$ the two following vectors:

$$
\begin{aligned}
D_{1} & =\left(\sigma_{0}-\sigma_{m}\right)\left[\int_{0}^{1} f(y) d y n_{\mathcal{C}_{0}}+\int_{0}^{1} A^{0}(f(y), y) d y\right] \\
& +\left(\sigma_{m}-\sigma_{1}\right) \int_{0}^{1} A^{0}(0, y) d y-\sigma_{0} a^{0}
\end{aligned}
$$

and respectively

$$
D_{2}=\left(\sigma_{m}-\sigma_{0}\right)\left[\int_{0}^{1} A^{0}(f(y), y) f^{\prime}(y) d y-\int_{0}^{1} f(y) d y\left(\begin{array}{c}
0 \\
1
\end{array}\right)\right] .
$$

Let $u^{0}$ be the unique solution of the following problem:

$$
\begin{aligned}
& \Delta u^{0}=0, \text { in } \mathcal{D}^{0} \cup \mathcal{D}^{1}, \\
& \left.\sigma_{0} \partial_{n} u^{0}\right|_{\Gamma^{+}}=\left.\sigma_{1} \partial_{n} u^{0}\right|_{\Gamma^{-}}, \\
& \left.u^{0}\right|_{\Gamma^{+}}=\left.u^{0}\right|_{\Gamma^{-}}, \\
& \left.u^{0}\right|_{\partial \Omega}=g,
\end{aligned}
$$

and define $u^{1}$ by:

$$
\begin{aligned}
& \Delta u^{1}=0, \text { in } \mathcal{D}^{0} \cup \mathcal{D}^{1}, \\
& \left.\sigma_{0} \partial_{n} u^{1}\right|_{\Gamma^{+}}=\left.\sigma_{1} \partial_{n} u^{1}\right|_{\Gamma^{-}}-\kappa D_{1} \cdot\left(\begin{array}{c}
\left.\partial_{n} u^{0}\right|_{\Gamma^{+}} \\
\left.\partial_{t} u^{0}\right|_{\Gamma^{+}}
\end{array}\right)+D_{2} \cdot \partial_{t}\left(\begin{array}{c}
\left.\partial_{n} u^{0}\right|_{\Gamma_{+}} \\
\left.\partial_{t} u^{0}\right|_{\Gamma^{+}}
\end{array}\right), \\
& \left.u^{1}\right|_{\Gamma^{+}}=\left.u^{1}\right|_{\Gamma^{-}}+a^{0} \cdot\left(\begin{array}{c}
\left.\partial_{n} u^{0}\right|_{\Gamma+} \\
\left.\partial_{t} u^{0}\right|_{\Gamma^{+}}
\end{array}\right), \\
& \left.u^{1}\right|_{\partial \Omega}=0
\end{aligned}
$$

where $\partial_{t}$ and $\partial_{n}$ denote the tangential and the normal derivatives along $\Gamma$. 
Theorem 1.1. Suppose that $g$ belongs to $H^{7 / 2}(\partial \Omega)$. Let $W$ be

$$
W=u^{\varepsilon}-\left(u^{0}+\varepsilon u^{1}\right) .
$$

Then, for any domain $\omega_{0}$ and $\omega_{1}$ respectively compactly embedded in $\Omega \backslash \overline{\mathcal{D}^{1}}$ and in $\mathcal{D}^{1}$ and for any $\left.\left.s \in\right] 1,2\right]$ there exists $\varepsilon_{0}>0$ and $C>0$ such that for any $\varepsilon \in\left(0, \varepsilon_{0}\right)$ :

$$
\begin{aligned}
\|W\|_{H^{1}\left(\omega_{0}\right)} & \leq C \varepsilon^{1+1 / s}, \\
\|W\|_{H^{1}\left(\omega_{1}\right)} & \leq C \varepsilon^{1+1 / s} .
\end{aligned}
$$

Remark 1.2. It is possible to give a precise behavior of $u^{\varepsilon}$ in a neighborhood of $\Gamma$, with the help of boundary layer correctors (see Theorem 4.1).

Remark 1.3. Observe the influence of the curvature appears in the definition of $u^{1}$, while no curvature was present at the first order for a weakly oscillating thin layer [7]. Actually if no oscillation occurs in the thin layer, i.e. if $f$ is a constant, $A^{0}$ is independent on $y$ and equals:

$$
A^{0}=\left(\begin{array}{c}
A_{1}^{0} \\
A_{2}^{0}
\end{array}\right)
$$

where

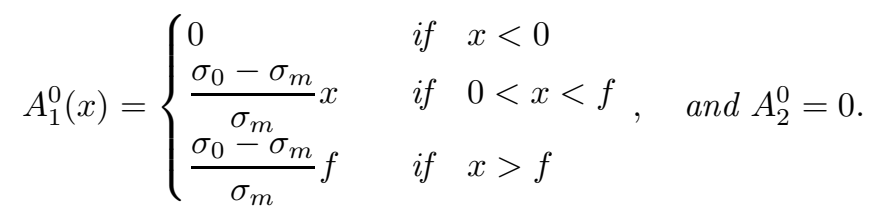

Then we easily obtain

$$
D_{1}=\left(\begin{array}{l}
0 \\
0
\end{array}\right), \quad D_{2}=\left(\begin{array}{c}
0 \\
\left(\sigma_{0}-\sigma_{m}\right) f
\end{array}\right) .
$$

hence we obtain the following approximate transmission conditions for $v^{1}$ :

$$
\begin{aligned}
& \Delta v^{1}=0, \text { in } \mathcal{O}^{1} \cup \mathcal{O}^{0}, \\
& \left.\sigma_{0} \partial_{n} v^{1}\right|_{\Gamma^{+}}=\left.\sigma_{1} \partial_{n} v^{1}\right|_{\Gamma^{-}}+\left.\left(\sigma_{0}-\sigma_{m}\right) f \partial_{t}^{2} v^{0}\right|_{\Gamma^{+}}, \\
& \left.v^{1}\right|_{\Gamma^{+}}=\left.v^{1}\right|_{\Gamma^{-}}+\left.\frac{\sigma_{0}-\sigma_{m}}{\sigma_{m}} f \partial_{n} v^{0}\right|_{\Gamma^{+}}, \\
& \left.v^{1}\right|_{\partial \Omega}=0, \text { on } \partial \Omega,
\end{aligned}
$$

where $\partial_{t}$ is the tangential derivative on $\Gamma$, which is the transmission conditions given in [8, 77].

\section{Preliminary analysis}

\subsection{The equivalent problem in a tubular neighborhood of $\Gamma$}

It is convenient to write problem (11) in a smooth tubular neighborhood of $\Gamma$. In the following, a normal $n$ to a closed smooth curve of $\Omega$ is the exterior normal 
vector to boundary of the domain enclosed by the curve. Let $\kappa$ be the curvature of $\Gamma$, and choose $d_{0}$ such that:

$$
0<d_{0}<\frac{1}{\|\kappa\|_{\infty}}
$$

Denote by $\Omega^{d_{0}}$ the open tubular neighborhood of $\Gamma$ at the distance $d_{0}$ :

$$
\Omega^{d_{0}}=\left\{z \in \mathbb{R}^{2}, \quad \operatorname{dist}(z, \Gamma)<d_{0}\right\} .
$$

Denote by $\Gamma_{-d_{0}}$ and $\Gamma_{d_{0}}$ the closed curves respectively defined by:

$$
\Gamma_{-d_{0}}=\partial \Omega^{d_{0}} \cap \mathcal{D}^{1}, \quad \Gamma_{d_{0}}=\partial \Omega^{d_{0}} \cap \mathcal{D}_{\varepsilon}^{0} .
$$

We now consider the following operators $\mathcal{L}_{0}, \mathcal{L}_{1}$ and $\mathscr{T}$ of Steklov-Poincaré type :

$$
\begin{aligned}
\mathcal{L}_{1} & : H^{1 / 2}\left(\Gamma_{-d_{0}}\right) \longrightarrow H^{-1 / 2}\left(\Gamma_{-d_{0}}\right), \\
\mathcal{L}_{0} & : H^{1 / 2}\left(\Gamma_{d_{0}}\right) \longrightarrow H^{-1 / 2}\left(\Gamma_{d_{0}}\right), \\
\mathscr{T} & : H^{1 / 2}(\partial \Omega) \longrightarrow H^{-1 / 2}\left(\Gamma_{d_{0}}\right)
\end{aligned}
$$

The operator $\mathcal{L}_{1}$ is defined by

$$
\forall \phi \in H^{1 / 2}\left(\Gamma_{-d_{0}}\right), \mathcal{L}_{1}(\phi)=\left.\frac{\partial u}{\partial n}\right|_{\Gamma_{-d_{0}}},
$$

where $u$ is the harmonic function in $\Omega \backslash \overline{\left(\mathcal{D}^{0} \cup \Omega^{d_{0}}\right)}$ equal to $\phi$ on $\Gamma_{-d_{0}}$. The operator $\mathcal{L}_{0}$ is defined by:

$$
\forall \phi \in H^{1 / 2}\left(\Gamma_{d_{0}}\right), \mathcal{L}_{0}(\phi)=\left.\frac{\partial u}{\partial n}\right|_{\Gamma_{d_{0}}}
$$

where $u$ is the harmonic function in $\Omega \backslash \overline{\left(\mathcal{D}^{1} \cup \Omega^{d_{0}}\right)}$ equal to $\phi$ on $\Gamma_{d_{0}}$ and vanishing on $\partial \Omega$. Similarly $\mathscr{T}$ equals:

$$
\forall \chi \in H^{1 / 2}(\partial \Omega), \mathscr{T}(\chi)=\left.\frac{\partial u}{\partial n}\right|_{\Gamma_{d_{0}}},
$$

where $u$ is the harmonic function in $\Omega \backslash \overline{\left(\mathcal{D}^{1} \cup \Omega^{d_{0}}\right)}$ equal to $\chi$ on $\partial \Omega$ and vanishing on $\Gamma_{d_{0}}$. Moreover the operators $\mathscr{T}, \mathcal{L}_{0}$ and $\mathcal{L}_{1}$ satisfies the following inequalities, for an $\varepsilon$-independent constant $C$ :

$$
\begin{gathered}
\forall g \in H^{1 / 2}(\partial \Omega), \quad|\mathscr{T} g|_{H^{-1 / 2}\left(\Gamma_{d_{0}}\right)} \leq C|g|_{H^{1 / 2}(\partial \Omega)}, \\
\forall u \in H^{1 / 2}\left(\Gamma_{d_{0}}\right), \quad\left|\mathcal{L}_{0} u\right|_{H^{-1 / 2}\left(\Gamma_{d_{0}}\right)} \leq C|u|_{H^{1 / 2}\left(\Gamma_{d_{0}}\right)}, \\
\forall u \in H^{1 / 2}\left(\Gamma_{-d_{0}}\right), \quad\left|\mathcal{L}_{1} u\right|_{H^{-1 / 2}\left(\Gamma_{-d_{0}}\right)} \leq C|u|_{H^{1 / 2}\left(\Gamma_{-d_{0}}\right)},
\end{gathered}
$$

and the coercivity inequalities:

$$
\begin{gathered}
\forall u \in H^{1 / 2}\left(\Gamma_{d_{0}}\right), \quad\left(\mathcal{L}_{0} u, u\right)_{L^{2}\left(\Gamma_{d_{0}}\right)} \geq C|u|_{H^{1 / 2}\left(\Gamma_{d_{0}}\right)}^{2}, \\
\forall u \in H^{1 / 2}\left(\Gamma_{-d_{0}}\right), \quad\left(\mathcal{L}_{1} u, u\right)_{L^{2}\left(\Gamma_{-d_{0}}\right)} \geq C|u|_{H^{1 / 2}\left(\Gamma_{-d_{0}}\right)}^{2} .
\end{gathered}
$$


Problem (1) is then equivalent to:

$$
\begin{aligned}
& \nabla .\left(\sigma \nabla u^{\varepsilon}\right)=0, \text { in } \Omega^{d_{0}}, \\
& \left.\partial_{n} u^{\varepsilon}\right|_{\Gamma_{d_{0}}}+\left.\mathcal{L}_{0} u^{\varepsilon}\right|_{\Gamma_{d_{0}}}=\mathscr{T} g, \text { on } \Gamma_{d_{0}}, \\
& \left.\partial_{n} u^{\varepsilon}\right|_{\Gamma_{-d_{0}}}+\left.\mathcal{L}_{1} u^{\varepsilon}\right|_{\Gamma_{-d_{0}}}=0, \text { on } \Gamma_{-d_{0}} .
\end{aligned}
$$

\subsection{The problem in local coordinates}

Denote by $\Phi$ the smooth diffeomorphism

$$
\forall(\eta, \theta) \in\left(-d_{0}, d_{0}\right) \times \mathbb{T}, \quad \Phi(\eta, \theta)=\Psi(\theta)+\eta n(\theta) .
$$

Since $d_{0}<1 /\|\kappa\|_{\infty}$, the open neighborhood of $\Gamma$ denoted by $\Omega^{d_{0}}$ might be parameterized as follows:

$$
\Omega^{d_{0}}=\left\{\Phi(\eta, \theta),(\eta, \theta) \in\left(-d_{0}, d_{0}\right) \times \mathbb{T}\right\}
$$

For $\varepsilon>0, \Omega^{d_{0}}$ is splitted into three subdomains $\Omega^{1}, \Omega_{\varepsilon}^{m}$ and $\Omega_{\varepsilon}^{0}$. These domains are defined with the help of the smooth 1-periodic function $f$ :

$$
\begin{aligned}
& \Omega^{1}=\left\{\Phi(\eta, \theta),(\eta, \theta) \in\left(-d_{0}, 0\right) \times \mathbb{T}\right\}, \\
& \Omega_{\varepsilon}^{m}=\{\Phi(\eta f(\theta / \varepsilon), \theta),(\eta, \theta) \in(0, \varepsilon) \times \mathbb{T}\}, \\
& \Omega_{\varepsilon}^{0}=\Omega^{d_{0}} \backslash\left(\overline{\Omega^{1} \cup \Omega_{\varepsilon}^{m}}\right) .
\end{aligned}
$$

Let $\mathcal{O}=\left(-d_{0}, d_{0}\right) \times \mathbb{T}$ and denote respectively by $\mathcal{O}^{1}, \mathcal{O}_{\varepsilon}^{m}$ and $\mathcal{O}_{\varepsilon}^{0}$ the following domains:

$$
\begin{aligned}
& \mathcal{O}^{1}=\left(-d_{0}, 0\right) \times \mathbb{T}, \\
& \mathcal{O}_{\varepsilon}^{m}=\{(\eta f(\theta / \varepsilon), \theta) \in(0, \varepsilon) \times \mathbb{T}\}, \\
& \mathcal{O}_{\varepsilon}^{0}=\mathcal{O} \backslash \overline{\mathcal{O}^{1} \cup \mathcal{O}_{\varepsilon}^{m}} .
\end{aligned}
$$

We also denote by $\Omega^{0}$ and $\mathcal{O}^{0}$ the respective domains $\Omega \backslash \Omega^{1}$ and $\mathcal{O} \backslash \mathcal{O}^{1}$. Define the following oscillating curve $\gamma_{\varepsilon}$ by

$$
\gamma_{\varepsilon}=\{(\varepsilon f(\theta / \varepsilon), \theta), \theta \in \mathbb{T}\}
$$

We denote by $\gamma^{s}=\{s\} \times \mathbb{T}$ for any $s \in \mathbb{R}$. The Laplacian operator written in $(\eta, \theta)$-coordinates equals:

$$
\Delta_{\eta, \theta}=\frac{1}{1+\eta \kappa(\theta)} \partial_{\eta}\left((1+\eta \kappa(\theta)) \partial_{\eta}\right)+\frac{1}{1+\eta \kappa(\theta)} \partial_{\theta}\left(\frac{1}{1+\eta \kappa(\theta)} \partial_{\theta}\right) .
$$

We also need the normal derivatives on $\Gamma$ and $\Gamma_{\varepsilon}$ in $(\eta, \theta)$-coordinates. In the following, the notation $\nabla_{\eta, \theta}$ denotes the derivative operator:

$$
\nabla_{\eta, \theta}=\left(\begin{array}{l}
\partial_{\eta} \\
\partial_{\theta}
\end{array}\right)
$$


Let $u$ be defined on $\Omega$, and define $v$ on $\left(-d_{0}, d_{0}\right) \times \mathbb{T}$ by

$$
\forall(\eta, \theta) \in\left(-d_{0}, d_{0}\right) \times \mathbb{T}, \quad v(\eta, \theta)=u \circ \Phi(\eta, \theta) .
$$

The following equalities hold, for $u$ enough regular:

$$
\begin{aligned}
& \left.\partial_{n} u\right|_{\Gamma}=\left.\partial_{\eta} v\right|_{\eta=0}, \\
& \left.\partial_{n} u\right|_{\Gamma_{d_{0}}}=\left.\partial_{\eta} v\right|_{\eta=d_{0}}, \\
& \left.\partial_{n} u\right|_{\Gamma_{-d_{0}}}=\left.\partial_{\eta} v\right|_{\eta=-d_{0}},
\end{aligned}
$$

and

$$
\begin{aligned}
\left.\partial_{n} u\right|_{\Gamma_{\varepsilon}} & =\frac{1}{\sqrt{(1+\varepsilon \kappa f(\theta / \varepsilon))^{2}+\left(f^{\prime}(\theta / \varepsilon)\right)^{2}}}\left[(1+\varepsilon \kappa f(\theta / \varepsilon)) \partial_{\eta} v\right. \\
& \left.-\frac{f^{\prime}(\theta / \varepsilon)}{1+\varepsilon \kappa f(\theta / \varepsilon)} \partial_{\theta} v\right]\left.\right|_{\gamma_{\varepsilon}} .
\end{aligned}
$$

Moreover, we define the bounded linear operators $\Lambda_{0}, T: H^{1 / 2}\left(\gamma^{d_{0}}\right) \rightarrow H^{-1 / 2}\left(\gamma^{d_{0}}\right)$ and $\Lambda_{1}: H^{1 / 2}\left(\gamma^{-d_{0}}\right) \rightarrow H^{-1 / 2}\left(\gamma^{-d_{0}}\right)$ as follows:

$$
\begin{aligned}
<\Lambda_{0} \varphi, \psi>=<\mathcal{L}_{0}\left(\varphi \circ \Phi^{-1}\right), \psi \circ \Phi^{-1}>, & \forall \varphi, \psi \in H^{1 / 2}\left(\gamma^{d_{0}}\right), \\
<T \varphi, \psi>=<\mathscr{T}\left(\varphi \circ \Phi^{-1}\right), \psi \circ \Phi^{-1}>, & \forall \varphi, \psi \in H^{1 / 2}\left(\gamma^{d_{0}}\right), \\
<\Lambda_{1} \varphi, \psi>=<\mathcal{L}_{1}\left(\varphi \circ \Phi^{-1}\right), \psi \circ \Phi^{-1}>, & \forall \varphi, \psi \in H^{1 / 2}\left(\gamma^{-d_{0}}\right) .
\end{aligned}
$$

According to (3), there exists a constant $C>0 \varepsilon$-independent such that

$$
\begin{array}{ll}
\forall u \in H^{1 / 2}(\mathbb{T}), \quad\left(\Lambda_{0} u, u\right)_{L^{2}(\mathbb{T})} \geq C|u|_{H^{1 / 2}(\mathbb{T})}^{2}, \\
\forall u \in H^{1 / 2}(\mathbb{T}), \quad\left(\Lambda_{1} u, u\right)_{L^{2}(\mathbb{T})} \geq C|u|_{H^{1 / 2}(\mathbb{T})}^{2} .
\end{array}
$$

It is convenient to denote by $\left.\partial_{n}^{\Phi} v\right|_{\gamma_{\varepsilon}}$ the following quantity:

$$
\begin{aligned}
\left.\partial_{n}^{\Phi} v\right|_{\gamma_{\varepsilon}} & =\frac{1}{\sqrt{(1+\varepsilon \kappa f(\theta / \varepsilon))^{2}+\left(f^{\prime}(\theta / \varepsilon)\right)^{2}}}\left[(1+\varepsilon \kappa f(\theta / \varepsilon)) \partial_{\eta} v\right. \\
& \left.-\frac{f^{\prime}(\theta / \varepsilon)}{1+\varepsilon \kappa f(\theta / \varepsilon)} \partial_{\theta} v\right]\left.\right|_{\gamma_{\varepsilon}} .
\end{aligned}
$$

With these notations, we may write our initial problem (1) in local coordinates. Denoting by $v^{\varepsilon}$ the solution to problem (1) in $(\eta, \theta)$-coordinates:

$$
v^{\varepsilon}=u^{\varepsilon} \circ \Phi,
$$

$v^{\varepsilon}$ is continuous and satisfies:

$$
\begin{aligned}
& \Delta_{\eta, \theta} v^{\varepsilon}=0, \text { in } \mathcal{O}^{1} \cup \mathcal{O}_{\varepsilon}^{m} \cup \mathcal{O}_{\varepsilon}^{0}, \\
& \left.\left(1+d_{0} \kappa\right) \partial_{\eta} v^{\varepsilon}\right|_{\eta=d_{0}}+\left.\Lambda_{0} v^{\varepsilon}\right|_{\eta=d_{0}}=\mathscr{T} g, \\
& \left.\left(1-d_{0} \kappa\right) \partial_{\eta} v^{\varepsilon}\right|_{\eta=-d_{0}}-\left.\Lambda_{1} v^{\varepsilon}\right|_{\eta=-d_{0}}=0,
\end{aligned}
$$


with the following transmission:

$$
\begin{aligned}
& \left.\sigma_{0} \partial_{n}^{\Phi} v^{\varepsilon}\right|_{\gamma_{\varepsilon}^{+}}=\left.\sigma_{m} \partial_{n}^{\Phi} v^{\varepsilon}\right|_{\gamma_{\varepsilon}^{-}}, \\
& \left.\sigma_{m} \partial_{\eta} v^{\varepsilon}\right|_{\eta=0^{+}}=\left.\sigma_{1} \partial_{\eta} v^{\varepsilon}\right|_{\eta=0^{-}}
\end{aligned}
$$

\subsection{Preliminary result}

Denote by $\boldsymbol{C}$ the infinite cylinder $\mathbb{R} \times \mathbb{T}$. Split $\boldsymbol{C}$ into three subdomains $Y_{0}, Y_{m}$ and $Y_{1}$. The subdomains are defined by:

$$
\begin{aligned}
Y_{1} & =(-\infty, 0) \times \mathbb{T}, \\
Y_{m} & =\{(\eta f(\theta), \theta), \forall(\eta, \theta) \in(0,1) \times \mathbb{T}\}, \\
Y_{0} & =\mathbb{R} \times \mathbb{T} \backslash\left(\overline{Y_{1} \cup Y_{m}}\right) .
\end{aligned}
$$

The closed curves $\mathcal{C}_{1}$ and $\mathcal{C}_{0}$ are defined by:

$$
\begin{aligned}
& \mathcal{C}_{0}=\{0\} \times \mathbb{T}, \\
& \mathcal{C}_{1}=\{(f(y), y), \forall y \in \mathbb{T}\} ;
\end{aligned}
$$

$\mathcal{C}_{1}$ and $\mathcal{C}_{0}$ have the trigonometric orientation. Remember that the exterior normals to the respective curves $\mathcal{C}_{0}$ and $\mathcal{C}_{1}$, which are respectively denoted by $n_{\mathcal{C}_{0}}$ and by $n_{\mathcal{C}_{1}}$ are defined by (2).

We also introduce the piecewise constant function $\tilde{\sigma}: \boldsymbol{C} \rightarrow \mathbb{R}$ by

$$
\tilde{\sigma}(x)=\left\{\begin{array}{llc}
\sigma_{1} & \text { in } Y_{1} \\
\sigma_{m} & \text { in } Y_{m} \\
\sigma_{0} & \text { in } Y_{0}
\end{array}\right.
$$

Notation 2.1. In this paper, we use the following notations.

- $\mathcal{S}_{\text {per }}(\mathbb{R} \times \mathbb{T})$ is the space of periodic square-integrable functions in the second variable which decay exponentially when the first variable goes to infinity.

- For any function $g \in L^{2}(\mathbb{T})$ (respectively $g \in L^{2}(\mathbb{R} \times \mathbb{T})$ ) we denote $\left\{\hat{g}_{k}\right\}_{k \in \mathbb{Z}}$ (respectively $\left\{\hat{g}_{k}(x)\right\}_{k \in \mathbb{Z}}$ ) the coefficients of the Fourier expansion of $g$ (respectively $g(x, \cdot))$, that is

$$
g(y)=\sum_{k \in \mathbb{Z}} \widehat{g}_{k} e^{2 \pi \mathrm{i} k y} \quad\left(\text { respectively } \quad g(x, y)=\sum_{k \in \mathbb{Z}} \widehat{g}_{k}(x) e^{2 \pi \mathrm{i} k y}\right)
$$

Lemma 2.2. Let $F$ belong to $\mathcal{S}_{\text {per }}(\mathbb{R} \times \mathbb{T})$ and suppose that there exists $M>\sup _{y \in \mathbb{T}}|f(y)|, C>0, \delta_{0} \in\left[0,2 \pi\left[\right.\right.$ and a family $\left\{p_{k}(x)\right\}_{k \in \mathbb{Z}}$ of regular functions defined on $|x|>M$ satisfying

$$
\begin{aligned}
& \left|p_{k}(x)\right| \leq C e^{\delta_{0}|k|(x-M)} \quad \text { for } x>M, \\
& \left|p_{k}(x)\right| \leq C e^{-\delta_{0}|k|(x+M)} \quad \text { for } x<-M,
\end{aligned}
$$


such that $F$ has the following Fourier expansion on $|x|>M$ :

$$
\begin{gathered}
F(x, y)=\sum_{k \in \mathbb{Z}^{*}} \widehat{F}_{k}(M) p_{k}(x) e^{-2 \pi|k|(x-M)} e^{2 \pi \mathrm{i} k y}, \quad \forall x \geq M, \\
F(x, y)=\sum_{k \in \mathbb{Z}^{*}} \widehat{F}_{k}(-M) p_{k}(x) e^{2 \pi|k|(x+M)} e^{2 \pi \mathrm{i} k y}, \quad \forall x \leq-M .
\end{gathered}
$$

Let $\varphi$ and $\psi$ be two smooth functions respectively defined on $\mathcal{C}_{0}$ and $\mathcal{C}_{1}$ such that:

$$
\int_{\mathcal{C}_{0}} \varphi(\sigma) \mathrm{d} \sigma+\int_{\mathcal{C}_{1}} \psi(\sigma) \mathrm{d} \sigma+\int_{\mathbb{R} \times \mathbb{T}} \tilde{\sigma}(x, y) F(x, y) \mathrm{d} x \mathrm{~d} y=0 .
$$

Then, there exists a unique couple $(\alpha, a)$, where $\alpha$ is a continuous function and a is a constant, such that:

$$
\begin{aligned}
& \Delta \alpha=F, \text { in } Y_{1} \cup Y_{m} \cup Y_{0}, \\
& \left.\sigma_{0} \partial_{n} \alpha\right|_{\mathcal{C}_{1}^{+}}=\left.\sigma_{m} \partial_{n} \alpha\right|_{\mathcal{C}_{1}^{-}}+\psi, \\
& \left.\sigma_{m} \partial_{n} \alpha\right|_{\mathcal{C}_{0}^{+}}=\left.\sigma_{1} \partial_{n} \alpha\right|_{\mathcal{C}_{0}^{-}}+\varphi, \\
& \alpha \rightarrow_{x \rightarrow-\infty} 0, \alpha \rightarrow_{x \rightarrow+\infty} a .
\end{aligned}
$$

Moreover, $\alpha$ decays exponentially for $x$ tending to $-\infty$ and $\alpha-a$ decays exponentially for $x$ tending to $+\infty$.

Proof. To prove this lemma, we rewrite equivalently problem (9) in the finite strip $[-M, M] \times \mathbb{T}$. The solution $\alpha$ can be written as a Fourier expansion:

$$
\alpha(x, y)=\sum_{k \in \mathbb{Z}} \widehat{\alpha}_{k}(x) e^{2 \pi \mathrm{i} k y} .
$$

Replacing in the equations (9) and taking into account the hypothesis on $F$ we find

$$
\begin{aligned}
\widehat{\alpha}_{0}(x) & =\widehat{\alpha}_{0}(M) \quad \text { for } x \geq M \\
\widehat{\alpha}_{k}(x) & =\left(\widehat{\alpha}_{k}(M)+\frac{\widehat{F}_{k}(M)}{4 \pi|k|} \int_{M}^{+\infty} p_{k}(t) e^{-4 \pi|k|(t-M)} d t\right. \\
& \left.-\frac{\widehat{F}_{k}(M)}{4 \pi|k|} \int_{M}^{x} p_{k}(t) d t\right) e^{-2 \pi|k|(x-M)} \\
& -\frac{\widehat{F}_{k}(M)}{4 \pi|k|} \int_{x}^{+\infty} p_{k}(t) e^{-4 \pi|k|(t-M)} d t \cdot e^{2 \pi|k|(x-M)} \quad \text { for } x \geq M, k \neq 0
\end{aligned}
$$

and respectively

$$
\begin{aligned}
\widehat{\alpha}_{0}(x) & =\widehat{\alpha}_{0}(-M) \quad \text { for } x \leq-M \\
\widehat{\alpha}_{k}(x) & =\left(\widehat{\alpha}_{k}(-M)+\frac{\widehat{F}_{k}(-M)}{4 \pi|k|} \int_{-\infty}^{-M} p_{k}(t) e^{4 \pi|k|(t+M)} d t\right. \\
& \left.-\frac{\widehat{F}_{k}(-M)}{4 \pi|k|} \int_{x}^{-M} p_{k}(t) d t\right) e^{2 \pi|k|(x+M)} \\
& -\frac{\widehat{F}_{k}(-M)}{4 \pi|k|} \int_{-\infty}^{x} p_{k}(t) e^{4 \pi|k|(t+M)} d t \cdot e^{-2 \pi|k|(x+M)} \quad \text { for } x \leq-M, k \neq 0
\end{aligned}
$$


Let us now introduce the operators $\mathcal{Q}: H^{1 / 2}(\mathbb{T}) \rightarrow H^{-1 / 2}(\mathbb{T})$ and $\mathscr{R}_{M}, \mathscr{R}_{-M}: H^{-1}(\mathbb{T}) \rightarrow L^{2}(\mathbb{T})$ by

$$
\begin{aligned}
& \forall u \in H^{1}(\mathbb{T}), \quad \mathcal{Q} u(y)=2 \pi \sum_{k \in \mathbb{Z}^{*}}|k| \widehat{u}_{k} e^{\mathrm{i} k y} \\
& \forall u \in H^{-1}(\mathbb{T}), \quad \mathscr{R}_{M} u(y)=\sum_{k \in \mathbb{Z}^{*}}\left(\int_{M}^{+\infty} p_{k}(t) e^{-4 \pi|k|(t-M)} \mathrm{d} t\right) \widehat{u}_{k} e^{2 \pi \mathrm{i} k y}, \\
& \forall u \in H^{-1}(\mathbb{T}), \quad \mathscr{R}_{-M} u(y)=\sum_{k \in \mathbb{Z}^{*}}\left(\int_{-\infty}^{-M} p_{k}(t) e^{4 \pi|k|(t+M)} \mathrm{d} t\right) \widehat{u}_{k} e^{2 \pi \mathrm{i} k y} .
\end{aligned}
$$

Due to hypothesis (8) these operators are well defined. We infer the following mixed boundary condition on $x=M$ :

$$
\left.\partial_{x} \alpha\right|_{x=M}+\left.\mathcal{Q} \alpha\right|_{x=M}=-\left.\mathscr{R}_{M} F\right|_{x=M} .
$$

Similarly, we obtain in $x=-M$

$$
\left.\partial_{x} \alpha\right|_{x=-M}-\left.\mathcal{Q} \alpha\right|_{x=-M}=\left.\mathscr{R}_{-M} F\right|_{x=-M} .
$$

Problem (9) is equivalent to the following problem, written in the strip $[-M, M] \times$ T:

$$
\begin{aligned}
& \Delta \alpha=F, \text { in }\left(Y_{1} \cup Y_{m} \cup Y_{0}\right) \cap[-M, M] \times \mathbb{T}, \\
& \left.\sigma_{0} \partial_{n} \alpha\right|_{\mathcal{C}_{1}^{+}}=\left.\sigma_{m} \partial_{n} \alpha\right|_{\mathcal{C}_{1}^{-}}+\psi \\
& \left.\sigma_{m} \partial_{n} \alpha\right|_{\mathcal{C}_{0}^{+}}=\left.\sigma_{1} \partial_{n} \alpha\right|_{\mathcal{C}_{0}^{-}}+\varphi
\end{aligned}
$$

with the mixed boundary conditions

$$
\begin{aligned}
& \left.\partial_{x} \alpha\right|_{x=M}+\left.\mathcal{Q} \alpha\right|_{x=M}=-\left.\mathscr{R}_{M} F\right|_{x=M}, \\
& \left.\partial_{x} \alpha\right|_{x=-M}-\left.\mathcal{Q} \alpha\right|_{x=-M}=\left.\mathscr{R}_{-M} F\right|_{x=-M} .
\end{aligned}
$$

The variationnal formulation of (13) is then:

$$
\forall v \in H^{1}([-M, M] \times \mathbb{T}), \quad \mathscr{A}(\alpha, v)=\mathscr{B}(v),
$$

where $\mathscr{A}$ and $\mathscr{B}$ are given by

$$
\begin{aligned}
\mathscr{A}(u, v) & =\int_{[-M, M] \times \mathbb{T}} \tilde{\sigma} \nabla \alpha . \nabla v \mathrm{~d} x \mathrm{~d} y+\sigma_{0}<\left.\mathcal{Q} \alpha\right|_{x=M},\left.v\right|_{x=M}> \\
& +\sigma_{1}<\left.\mathcal{Q} \alpha\right|_{x=-M},\left.v\right|_{x=-M}>, \\
\mathscr{B}(v) & =-\int_{[-M, M] \times \mathbb{T}} \tilde{\sigma} F(x, y) v(x, y) \mathrm{d} x \mathrm{~d} y-\int_{\mathcal{C}_{0}} \phi(\sigma) v(\sigma) \mathrm{d} \sigma-\int_{\mathcal{C}_{1}} \psi(\sigma) v(\sigma) \mathrm{d} \sigma \\
- & \sigma_{0} \int_{\mathbb{T}} \mathscr{R}_{M} F(M, y) v(M, y) \mathrm{d} y-\sigma_{1} \int_{\mathbb{T}} \mathscr{R}_{-M} F(-M, y) v(-M, y) \mathrm{d} y .
\end{aligned}
$$

The continuity of $\mathscr{B}$ on $H^{1}([-M, M] \times \mathbb{T})$ and of $\mathscr{A}$ on $\left(H^{1}([-M, M] \times \mathbb{T})\right)^{2}$ easily comes from calculations in the Fourier variable. From Poincaré inequality 
we also deduce that $\mathscr{A}$ is coercive on $H^{1}([-M, M] \times \mathbb{T}) / \mathbb{R}$.

On the other hand, from the hypothesis on $F$ we deduce

$$
\int_{[M,+\infty[\times \mathbb{T}} F(x, y) d y d x=\int_{M}^{\infty}\left(\int_{\mathbb{T}} F(x, y) d y\right) d x=0,
$$

and similary

$$
\int_{]-\infty,-M] \times \mathbb{T}} F(x, y) d y d x=0 .
$$

We also have

$$
\int_{\mathbb{T}} \mathscr{R}_{M} F(M, y) d y=\int_{\mathbb{T}} \mathscr{R}_{-M} F(-M, y) d y=0
$$

which gives

$$
\mathscr{B}(1)=0 \text {. }
$$

So we have an unique solution of (14) up to an additive constant and we can choose this constant such that

$$
\int_{\mathbb{T}} \alpha(-M, y) d y=\widehat{\alpha}_{0}(-M)=0
$$

We then have

$$
a=\widehat{\alpha}_{0}(M)=\int_{\mathbb{T}} \alpha(M, y) d y
$$

The exponential decay of $\alpha-a$ in $+\infty$ and $\alpha$ in $-\infty$ comes from the expressions of $\widehat{\alpha}_{k}(x)$ for $k \neq 0$.

Remark 2.3. Suppose that for $|x|$ large enough $F$ equals $F(x, y)=F_{1}(x, y) F_{2}(x)$ where $F_{1}$ is an harmonic function and $F_{2}$ is such that

$$
\exists C>0 \text { and } \delta_{0} \in\left[0,2 \pi\left[\text { such that }\left|F_{2}(x)\right| \leq C e^{\delta_{0}|x|}, \forall x\right.\right.
$$

(for exemple if $F_{2}$ is a polynom). Then $F$ satisfies obviously the hypothesis of Lemma 2.2.

\section{Formal asymptotics}

\subsection{Zeroth order approximation}

Let $v^{0}$ be the continuous "background" solution defined by:

$$
\begin{aligned}
& \Delta_{\eta, \theta} v^{0}=0, \text { in } \mathcal{O}^{1} \cup \mathcal{O}^{0}, \\
& \left.\left(1+d_{0} \kappa\right) \partial_{\eta} v^{0}\right|_{\eta=d_{0}}+\left.\Lambda_{0} v^{0}\right|_{\eta=d_{0}}=\mathscr{T} g, \\
& \left.\left(1-d_{0} \kappa\right) \partial_{\eta} v^{0}\right|_{\eta=-d_{0}}-\left.\Lambda_{1} v^{0}\right|_{\eta=-d_{0}}=0, \\
& \left.\sigma_{0} \partial_{\eta} v^{0}\right|_{\eta=0^{+}}=\left.\sigma_{1} \partial_{\eta} v^{0}\right|_{\eta=0^{-}} .
\end{aligned}
$$

A classical regularity result implies that for $g \in H^{s}(\partial \Omega)$, the potential $v^{0}$ belongs to $H^{1}(\Omega)$ and moreover it has $H^{s+1 / 2}$-regularity in $\mathcal{O}^{1}$ and in $\mathcal{O}^{0}$. In this section, we suppose that $g$ is as regular as necessary so that the involved 
quantities are well-defined. Rigorous proof of the formal asymptotic expansion is performed in the next section.

Denote by $w^{0}$ the difference between the exact solution $v^{\varepsilon}$ and $v^{0}$. This continuous function is the solution of the following problem:

$$
\begin{aligned}
& \Delta_{\eta, \theta} w^{0}=0, \text { in } \mathcal{O}^{1} \cup \mathcal{O}^{0}, \\
& \left.\left(1+d_{0} \kappa\right) \partial_{\eta} w^{0}\right|_{\eta=d_{0}}+\left.\Lambda_{0} w^{0}\right|_{\eta=d_{0}}=0, \\
& \left.\left(1-d_{0} \kappa\right) \partial_{\eta} w^{0}\right|_{\eta=-d_{0}}-\left.\Lambda_{1} w^{0}\right|_{\eta=-d_{0}}=0, \\
& \left.\sigma_{0} \partial_{n}^{\Phi} w^{0}\right|_{\gamma_{\varepsilon}^{+}}=\left.\sigma_{m} \partial_{n}^{\Phi} w^{0}\right|_{\gamma_{\varepsilon}^{-}}+\left.\left(\sigma_{m}-\sigma_{0}\right) \partial_{n}^{\Phi} v^{0}\right|_{\gamma_{\varepsilon}}, \\
& \left.\sigma_{m} \partial_{\eta} w^{0}\right|_{\eta=0^{+}}=\left.\sigma_{1} \partial_{\eta} w^{0}\right|_{\eta=0^{-}}+\left.\left(\sigma_{0}-\sigma_{m}\right) \partial_{\eta} v^{0}\right|_{\eta=0^{+}}, \\
& \left.w^{0}\right|_{\partial \Omega}=0, \text { on } \partial \Omega .
\end{aligned}
$$

Since we are interested in the derivation up to the 1 - order terms, we throw away all the terms, which are a priori of order smaller than $\varepsilon^{2}$. This approximation will be rigorously justified in the next section.

Using the explicit expression of the normal derivative on $\gamma_{\varepsilon}$, we infer:

$$
\begin{aligned}
\left.\partial_{n}^{\Phi} v^{0}\right|_{\gamma_{\varepsilon}} & =\frac{1-\varepsilon \kappa f(\theta / \varepsilon) /\left(1+\left(f^{\prime}(\theta / \varepsilon)\right)^{2}\right)+O\left(\varepsilon^{2}\right)}{\sqrt{1+\left[f^{\prime}(\theta / \varepsilon)\right]^{2}}}\left[\left.(1+\varepsilon \kappa f(\theta / \varepsilon)) \partial_{\eta} v^{0}\right|_{\gamma_{\varepsilon}}\right. \\
& \left.-\left.f^{\prime}(\theta / \varepsilon)\left(1-\varepsilon \kappa f(\theta / \varepsilon)+O\left(\varepsilon^{2}\right)\right) \partial_{\theta} v^{0}\right|_{\gamma_{\varepsilon}}\right]
\end{aligned}
$$

Taylor expansion in the $\eta$-variable implies:

$$
\begin{aligned}
& \left.\partial_{\eta} v^{0}\right|_{\gamma_{\varepsilon}}=\left.\partial_{\eta} v^{0}\right|_{\eta=0^{+}}+\left.\varepsilon f(\theta / \varepsilon) \partial_{\eta}^{2} v^{0}\right|_{\eta=0^{+}}+O\left(\varepsilon^{2}\right), \\
& \left.\partial_{\theta} v^{0}\right|_{\gamma_{\varepsilon}}=\left.\partial_{\theta} v^{0}\right|_{\eta=0^{+}}+\left.\varepsilon f(\theta / \varepsilon) \partial_{\eta, \theta^{2}}^{2} v^{0}\right|_{\eta=0^{+}}+O\left(\varepsilon^{2}\right) .
\end{aligned}
$$

From the above equalities, we deduce

$$
\begin{aligned}
\left.\partial_{n}^{\Phi} v^{0}\right|_{\gamma_{\varepsilon}}= & \left.\frac{1}{\sqrt{1+\left(f^{\prime}\right)^{2}}} \partial_{\eta} v^{0}\right|_{\eta=0^{+}}-\left.\frac{f^{\prime}}{\sqrt{1+\left(f^{\prime}\right)^{2}}} \partial_{\theta} v^{0}\right|_{\eta=0^{+}}+ \\
& \varepsilon\left\{\frac { \kappa f } { \sqrt { 1 + ( f ^ { \prime } ) ^ { 2 } } } \left[\left.\left(1-\frac{1}{1+\left(f^{\prime}\right)^{2}}\right) \partial_{\eta} v^{0}\right|_{\eta=0^{+}}\right.\right. \\
& \left.+\left.\left(1+\frac{1}{1+\left(f^{\prime}\right)^{2}}\right) \partial_{\theta} v^{0}\right|_{\eta=0^{+}}\right]+\left.\frac{f}{\sqrt{1+\left(f^{\prime}\right)^{2}}} \partial_{\eta}^{2} v^{0}\right|_{\eta=0^{+}} \\
& \left.-\left.\frac{f^{\prime} f}{\sqrt{1+\left(f^{\prime}\right)^{2}}} \partial_{\eta \theta}^{2} v^{0}\right|_{\eta=0^{+}}\right\}+O\left(\varepsilon^{2}\right),
\end{aligned}
$$

where $f$ and $f^{\prime}$ are taken in $\frac{\theta}{\varepsilon}$. Since

$$
\left.\partial_{\eta}^{2} v^{0}\right|_{\eta=0^{+}}=-\left.\kappa \partial_{\eta} v^{0}\right|_{\eta=0^{+}}-\left.\partial_{\theta}^{2} v^{0}\right|_{\eta=0^{+}},
$$

denoting by $n_{\mathcal{C}_{1}}^{\perp}$ the following vector:

$$
n_{\mathcal{C}_{1}}^{\perp}=\frac{1}{\sqrt{1+\left(f^{\prime}\right)^{2}}}\left(\begin{array}{c}
f^{\prime} \\
1
\end{array}\right),
$$


we infer:

$$
\begin{aligned}
{\left[\sigma \partial_{n}^{\Phi} v^{0}\right]_{\gamma_{\varepsilon}}=} & \left.\left(\sigma_{0}-\sigma_{m}\right) n_{\mathcal{C}_{1}}(\theta / \varepsilon) \cdot \nabla_{\eta, \theta} v^{0}\right|_{\eta=0^{+}} \\
& +\varepsilon\left(\sigma_{0}-\sigma_{m}\right)\left\{\kappa f ( \theta / \varepsilon ) \left(\frac{n_{\mathcal{C}_{0}}(\theta / \varepsilon)}{\sqrt{1+\left(f^{\prime}(\theta / \varepsilon)\right)^{2}}}\right.\right. \\
& \left.-\frac{2+\left(f^{\prime}(\theta / \varepsilon)\right)^{2}}{1+\left(f^{\prime}(\theta / \varepsilon)\right)^{2}} n_{\mathcal{C}_{1}}(\theta / \varepsilon)\right)\left.\cdot \nabla_{\eta, \theta} v^{0}\right|_{\eta=0^{+}} \\
& \left.-\left.f(\theta / \varepsilon) n_{\mathcal{C}_{1}}^{\perp}(\theta / \varepsilon) \cdot \partial_{\theta} \nabla_{\eta, \theta} v^{0}\right|_{\eta=0^{+}}\right\}+O\left(\varepsilon^{2}\right) .
\end{aligned}
$$

Observe also that:

$$
\left[\sigma \partial_{n} v^{0}\right]_{\eta=0}=\left.\left(\sigma_{0}-\sigma_{m}\right) n_{\mathcal{C}_{0}}(\theta / \varepsilon) \cdot \nabla_{\eta, \theta} v^{0}\right|_{\eta=0^{+}} .
$$

Define now the vector field $A^{0}$ from which we will define the boundary layer corrector of order 0 . Since

$$
\int_{\mathcal{C}_{1}} n_{\mathcal{C}_{1}}(\sigma) \mathrm{d} \sigma-\int_{\mathcal{C}_{0}} n_{\mathcal{C}_{0}}(\sigma) \mathrm{d} \sigma=\left(\begin{array}{l}
0 \\
0
\end{array}\right),
$$

and according to lemma 2.2, there exists a unique couple $\left(A^{0}, a^{0}\right)$ (where $A^{0}$ is a continuous vector field and $a^{0}$ a constant vector) such that

$$
\begin{aligned}
& \Delta A^{0}=0, \text { in } \mathbb{R} \times \mathbb{T}, \\
& \left.\sigma_{0} \partial_{n} A^{0}\right|_{\mathcal{C}_{1}^{+}}-\left.\sigma_{m} \partial_{n} A^{0}\right|_{\mathcal{C}_{1}^{-}}=\left(\sigma_{m}-\sigma_{0}\right) n_{\mathcal{C}_{1}}, \\
& \left.\sigma_{m} \partial_{n} A^{0}\right|_{\mathcal{C}_{0}^{+}}-\left.\sigma_{1} \partial_{n} A^{0}\right|_{\mathcal{C}_{0}^{-}}=-\left(\sigma_{m}-\sigma_{0}\right) n_{\mathcal{C}_{0}}, \\
& A^{0} \rightarrow_{x \rightarrow-\infty} 0, A^{0} \rightarrow_{x \rightarrow+\infty} a^{0} .
\end{aligned}
$$

We define now the boundary layer corrector of order $0, v_{B L}^{0}$ on $\mathcal{O}$ by:

$$
v_{B L}^{0}(\eta, \theta)=\left\{\begin{array}{l}
\left.\varepsilon\left(A^{0}(\eta / \varepsilon, \theta / \varepsilon)-a^{0}\right) \cdot \nabla_{\eta, \theta} v^{0}\right|_{\Gamma^{+}}, \text {if } \eta>0, \\
\left.\varepsilon A^{0}(\eta / \varepsilon, \theta / \varepsilon) \cdot \nabla_{\eta, \theta} v^{0}\right|_{\Gamma^{+}}, \text {if } \eta<0 .
\end{array}\right.
$$

Remark 3.1. Using the equations satisfied by $A^{0}$, we observe that $A^{0}$ decays exponentially for $x \rightarrow-\infty$ and similarly $A^{0}-a^{0}$ decays exponentially for $x \rightarrow$ $\infty$.

From the equality (15) with $v^{0}$ replaced by $v_{B L}^{0}$ we deduce

$$
\begin{aligned}
\left.\partial_{n}^{\Phi} v_{B L}^{0}\right|_{\gamma_{\varepsilon}} & =\left.\left(\frac{1}{\sqrt{1+\left(f^{\prime}\right)^{2}}} \frac{\partial A^{0}}{\partial x}-\frac{f^{\prime}}{\sqrt{1+\left(f^{\prime}\right)^{2}}} \frac{\partial A^{0}}{\partial y}\right) \cdot \nabla_{\eta, \theta} v^{0}\right|_{\eta=0^{+}} \\
& -\left.\varepsilon \frac{f^{\prime}}{\sqrt{1+\left(f^{\prime}\right)^{2}}}\left(A^{0}-a^{0}\right) \cdot \partial_{\theta} \nabla_{\eta, \theta} v^{0}\right|_{\eta=0^{+}} \\
& +\varepsilon \kappa f\left(\left[\left(1+\left(f^{\prime}\right)^{2}\right)^{-1 / 2}-\left(1+\left(f^{\prime}\right)^{2}\right)^{-3 / 2}\right] \frac{\partial A^{0}}{\partial x}\right. \\
& \left.+\left[\left(1+\left(f^{\prime}\right)^{2}\right)^{-1 / 2}+\left(1+\left(f^{\prime}\right)^{2}\right)^{-3 / 2}\right] f^{\prime} \frac{\partial A^{0}}{\partial y}\right)\left.\cdot \nabla_{\eta, \theta} v^{0}\right|_{\eta=0^{+}}+O\left(\varepsilon^{2}\right) .
\end{aligned}
$$

$\mathrm{RR} \mathrm{n}^{\circ} 6812$ 
Taking into account the fact that

$$
\frac{1}{\sqrt{1+\left(f^{\prime}\right)^{2}}}\left[\sigma \frac{\partial A^{0}}{\partial x}\right]_{\mathcal{C}_{1}}-\frac{f^{\prime}}{\sqrt{1+\left(f^{\prime}\right)^{2}}}\left[\sigma \frac{\partial A^{0}}{\partial y}\right]_{\mathcal{C}_{1}}=\left[\sigma \frac{\partial A^{0}}{\partial n}\right]_{\mathcal{C}_{1}},
$$

we obtain

$$
\begin{aligned}
{\left[\sigma \partial_{n}^{\Phi} v_{B L}^{0}\right]_{\gamma_{\varepsilon}} } & =\left.\left(\sigma_{m}-\sigma_{0}\right) n_{C_{1}} \cdot \nabla_{\eta, \theta} v^{0}\right|_{\eta=0^{+}} \\
& +\left.\varepsilon\left(\sigma_{m}-\sigma_{0}\right) \frac{f^{\prime}}{\sqrt{1+\left(f^{\prime}\right)^{2}}}\left(A^{0}-a^{0}\right) \cdot \partial_{\theta} \nabla_{\eta, \theta} v^{0}\right|_{\eta=0^{+}} \\
& +\varepsilon \kappa f\left(\frac{2}{\sqrt{1+\left(f^{\prime}\right)^{2}}}\left[\sigma \frac{\partial A^{0}}{\partial x}\right]_{\mathcal{C}_{1}}\right. \\
& \left.-\left(\sigma_{m}-\sigma_{0}\right) \frac{2+\left(f^{\prime}\right)^{2}}{1+\left(f^{\prime}\right)^{2}} n_{\mathcal{C}_{1}}\right)\left.\cdot \nabla_{\eta, \theta} v^{0}\right|_{\eta=0^{+}}+O\left(\varepsilon^{2}\right)
\end{aligned}
$$

Therefore, using (17)-(18) we infer

$$
\begin{aligned}
{\left[\sigma \partial_{n}^{\Phi}\left(v^{0}+v_{B L}^{0}\right)\right]_{\gamma_{\varepsilon}} } & =\left(\sigma_{0}-\sigma_{m}\right) \varepsilon\left\{\kappa \frac { f ( \theta / \varepsilon ) } { \sqrt { 1 + ( f ^ { \prime } ( \theta / \varepsilon ) ) ^ { 2 } } } \left(n_{\mathcal{C}_{0}}\right.\right. \\
& \left.+\frac{2}{\sigma_{0}-\sigma_{m}}\left[\sigma \frac{\partial A^{0}}{\partial x}\right]_{\mathcal{C}_{1}}\right)\left.\cdot \nabla_{\eta, \theta} v^{0}\right|_{\eta=0^{+}} \\
& \left.-\left(f n_{\mathcal{C}_{1}}^{\perp}+\frac{f^{\prime}}{\sqrt{1+\left(f^{\prime}\right)^{2}}}\left(A^{0}-a^{0}\right)\right) \cdot \partial_{\theta} \nabla_{\eta, \theta} v^{0}\right\}+O\left(\varepsilon^{2}\right) .
\end{aligned}
$$

On the other hand, for any $v$ regular enough we have:

$$
\Delta_{\eta, \theta} v=\partial_{\eta}^{2} v+\partial_{\theta}^{2} v+\left((1+\eta \kappa)^{-2}-1\right) \partial_{\theta}^{2} v+\frac{\kappa}{1+\eta \kappa} \partial_{\eta} v-\frac{\eta \kappa^{\prime}}{(1+\eta \kappa)^{3}} \partial_{\theta} v .
$$

Now from the equation satisfied by $A^{0}$ we obtain:

$$
\begin{aligned}
\Delta_{\eta, \theta} v_{B L}^{0}= & \left.2 \frac{\partial A^{0}}{\partial y} \cdot \partial_{\theta} \nabla_{\eta, \theta} v^{0}\right|_{\eta=0^{+}}-\frac{2 \eta \kappa+\eta^{2} \kappa^{2}}{(1+\eta \kappa)^{2}}\left(\left.\frac{1}{\varepsilon} \frac{\partial^{2} A^{0}}{\partial y^{2}} \cdot \nabla_{\eta, \theta} v^{0}\right|_{\eta=0^{+}}\right. \\
& \left.+\left.2 \frac{\partial A^{0}}{\partial y} \cdot \partial_{\theta} \nabla_{\eta, \theta} v^{0}\right|_{\eta=0^{+}}\right)+\left.\frac{\kappa}{1+\eta \kappa} \frac{\partial A^{0}}{\partial x} \cdot \nabla_{\eta, \theta} v^{0}\right|_{\eta=0^{+}} \\
& -\left.\frac{\eta \kappa^{\prime}}{(1+\eta \kappa)^{3}} \frac{\partial A^{0}}{\partial y} \cdot \nabla_{\eta, \theta} v^{0}\right|_{\eta=0^{+}}+O(\varepsilon),
\end{aligned}
$$

which gives using the equality $\eta=x \varepsilon$ :

$$
\Delta_{\eta, \theta} v_{B L}^{0}=\left.\kappa\left(\frac{\partial A^{0}}{\partial x}-2 x \frac{\partial^{2} A^{0}}{\partial y^{2}}\right) \cdot \nabla_{\eta, \theta} v^{0}\right|_{\eta=0^{+}}+\left.2 \frac{\partial A^{0}}{\partial y} \cdot \partial_{\theta} \nabla_{\eta, \theta} v^{0}\right|_{\eta=0^{+}}+\tilde{O}(\varepsilon),
$$

where $\tilde{O}(\varepsilon)$ denotes any function of order $\varepsilon$ when $|\eta| / \varepsilon$ is bounded and whith exponential decays for $|\eta| / \varepsilon$ tending to infinity. 


\subsection{First order approximation}

For a function $u$ defined in $\mathcal{O}^{0} \cup \mathcal{O}^{1}$, we denote by $\left.[u]\right|_{\gamma_{\varepsilon}}$ the jump of $u$ across $\gamma_{\varepsilon}$ :

$$
\left.[u]\right|_{\gamma_{\varepsilon}}=\left.u\right|_{\gamma_{\varepsilon}^{+}}-\left.u\right|_{\gamma_{\varepsilon}^{-}} .
$$

Using the equalities (17), (18) and (20) we deduce that the function $W_{0}$ defined by $W_{0}=v^{\varepsilon}-\left(v^{0}+v_{B L}^{0}\right)$ satisfies:

$$
\begin{aligned}
& \Delta_{\eta, \theta} W^{0}=\left.\kappa(\theta) G_{1}\left(\frac{\eta}{\varepsilon}, \frac{\theta}{\varepsilon}\right) \cdot \nabla_{\eta, \theta} v^{0}\right|_{\eta=0^{+}}+\left.G_{2}\left(\frac{\eta}{\varepsilon}, \frac{\theta}{\varepsilon}\right) \cdot \partial_{\theta} \nabla_{\eta, \theta} v^{0}\right|_{\eta=0^{+}}+\tilde{O}(\varepsilon), \\
& \left.\left(1+d_{0} \kappa\right) \partial_{\eta} W^{0}\right|_{\eta=d_{0}}+\left.\Lambda_{0} W^{0}\right|_{\eta=d_{0}}=g_{0+}^{\varepsilon}, \\
& \left.\left(1-d_{0} \kappa\right) \partial_{\eta} W^{0}\right|_{\eta=-d_{0}}-\left.\Lambda_{1} W^{0}\right|_{\eta=-d_{0}}=g_{0-}^{\varepsilon},
\end{aligned}
$$

with the following transmission conditions:

$$
\begin{aligned}
& \left.\sigma_{0} \partial_{n}^{\Phi} W^{0}\right|_{\gamma_{\varepsilon}^{+}}-\left.\sigma_{m} \partial_{n}^{\Phi} W^{0}\right|_{\gamma_{\varepsilon}^{-}}=\varepsilon\left[\left.\kappa(\theta) B_{1}(\theta / \varepsilon) \cdot \nabla_{\eta, \theta} v^{0}\right|_{\eta=0^{+}}\right. \\
& \left.+\left.B_{2}(\theta / \varepsilon) \cdot \partial_{\theta} \nabla_{\eta, \theta} v^{0}\right|_{\eta=0^{+}}\right], \\
& \left.\sigma_{m} \partial_{\eta} W^{0}\right|_{\eta=0^{+}}-\left.\sigma_{1} \partial_{\eta} W^{0}\right|_{\eta=0^{-}}=0,
\end{aligned}
$$

and

$$
\left.\left[W^{0}\right]\right|_{\gamma_{\varepsilon}}=0,\left.\quad\left[W^{0}\right]\right|_{\eta=0}=\left.\varepsilon a^{0} \cdot \nabla_{\eta, \theta} v^{0}\right|_{\eta=0^{+}},
$$

where $G_{1}, G_{2}, B_{1}$ and $B_{2}$ are given with the help of (20)-(19) by:

$$
\begin{aligned}
G_{1} & =-\left(\frac{\partial A^{0}}{\partial x}-2 x \frac{\partial^{2} A^{0}}{\partial y^{2}}\right), \\
G_{2} & =-2 \frac{\partial A^{0}}{\partial y} \\
B_{1} & =-\frac{f}{\sqrt{1+\left(f^{\prime}\right)^{2}}}\left(\left(\sigma_{0}-\sigma_{m}\right) n_{\mathcal{C}_{0}}+2\left[\sigma \frac{\partial A^{0}}{\partial x}\right]_{\mathcal{C}_{1}}\right), \\
B_{2} & =\left(\sigma_{0}-\sigma_{m}\right)\left(f n_{\mathcal{C}_{1}}^{\perp}+\frac{f^{\prime}}{\sqrt{1+\left(f^{\prime}\right)^{2}}}\left(A^{0}-a^{0}\right)\right),
\end{aligned}
$$

and

$$
\begin{aligned}
& g_{0+}^{\varepsilon}=-\left(1+d_{0} \kappa\right) \partial_{\eta} v_{B L}^{0}\left(\eta_{0}, \theta\right)-\Lambda_{0}\left(\left.v_{B L}^{0}\right|_{\eta=\eta_{0}}\right), \\
& g_{0-}^{\varepsilon}=-\left(1-d_{0} \kappa\right) \partial_{\eta} v_{B L}^{0}\left(-\eta_{0}, \theta\right)+\Lambda_{1}\left(\left.v_{B L}^{0}\right|_{\eta=-\eta_{0}}\right) .
\end{aligned}
$$

We now define the following vectors, for $k=1,2$ :

$$
D_{k}=\int_{\mathbb{R} \times \mathbb{T}} \tilde{\sigma} G_{k} d x d y+\int_{\mathcal{C}_{1}} B_{k} d \sigma,
$$

with $\tilde{\sigma}$ defined in (7). Simple calculus give

$$
\begin{aligned}
D_{1} & =\left(\sigma_{0}-\sigma_{m}\right)\left[\int_{0}^{1} f(y) d y n_{\mathcal{C}_{0}}+\int_{0}^{1} A^{0}(f(y), y) d y\right] \\
& +\left(\sigma_{m}-\sigma_{1}\right) \int_{0}^{1} A^{0}(0, y) d y-\sigma^{0} a^{0}
\end{aligned}
$$


and respectively

$$
D_{2}=\left(\sigma_{m}-\sigma_{0}\right)\left[\int_{0}^{1} A^{0}(f(y), y) f^{\prime}(y) d y-\int_{0}^{1} f(y) d y\left(\begin{array}{l}
0 \\
1
\end{array}\right)\right] .
$$

Define $v^{1}$ by:

$$
\begin{aligned}
& \Delta_{\eta, \theta} v^{1}=0, \text { in } \mathcal{O}^{1} \cup \mathcal{O}^{0} \\
& \left.\left(1+d_{0} \kappa\right) \partial_{\eta} v^{1}\right|_{\eta=d_{0}}+\left.\Lambda_{0} v^{1}\right|_{\eta=d_{0}}=0 \\
& \left.\left(1-d_{0} \kappa\right) \partial_{\eta} v^{1}\right|_{\eta=-d_{0}}-\left.\Lambda_{1} v^{1}\right|_{\eta=-d_{0}}=0
\end{aligned}
$$

with the following transmission conditions

$$
\begin{aligned}
& \left.\sigma_{0} \partial_{\eta} v^{1}\right|_{\eta=0^{+}}-\left.\sigma_{1} \partial_{\eta} v^{1}\right|_{\eta=0^{-}}=\left.\kappa D_{1} \cdot \nabla_{\eta, \theta} v\right|_{\eta=0^{+}} ^{0}+\left.D_{2} \cdot \partial_{\theta} \nabla_{\eta, \theta} v\right|_{\eta=0^{+}} ^{0}, \\
& \left.v^{1}\right|_{\eta=0^{+}}-\left.v^{1}\right|_{\eta=0^{-}}=\left.a^{0} \cdot\left(\nabla_{\eta, \theta} v^{0}\right)\right|_{\eta=0^{+}}
\end{aligned}
$$

Denote by $w^{1}$ the following quantity:

$$
w^{1}=W^{0}-\varepsilon v^{1} .
$$

Since we have

$$
\left.\partial_{n}^{\Phi} v^{1}\right|_{\gamma_{\varepsilon}}=\left.n_{\mathcal{C}_{1}} \cdot \nabla_{\eta, \theta} v^{1}\right|_{\eta=0^{+}}+O(\varepsilon),
$$

and since $\left.\partial_{\eta} v^{1}\right|_{\eta=0^{+}}=\left.n_{\mathcal{C}_{0}} \cdot \nabla_{\eta, \theta} v^{1}\right|_{\eta=0^{+}}$then $w^{1}$ satisfies:

$$
\begin{aligned}
& \Delta_{\eta, \theta} w^{1}=\left.\kappa(\theta) G_{1} \cdot \nabla_{\eta, \theta} v^{0}\right|_{\eta=0^{+}}+\left.G_{2} \cdot \partial_{\theta} \nabla_{\eta, \theta} v^{0}\right|_{\eta=0^{+}}+\tilde{O}(\varepsilon), \\
& \left.\left(1+d_{0} \kappa\right) \partial_{\eta} w^{1}\right|_{\eta=d_{0}}+\left.\Lambda_{0} w^{1}\right|_{\eta=d_{0}}=g_{0+}^{\varepsilon} \\
& \left.\left(1-d_{0} \kappa\right) \partial_{\eta} w^{1}\right|_{\eta=-d_{0}}-\left.\Lambda_{1} w^{1}\right|_{\eta=-d_{0}}=g_{0-}^{\varepsilon}
\end{aligned}
$$

with the following transmission conditions:

$$
\begin{aligned}
{\left[\sigma \partial_{n}^{\Phi} w^{1}\right]_{\gamma_{\varepsilon}} } & =\varepsilon\left(\left.\kappa(\theta) B_{1}(\theta / \varepsilon) \cdot \nabla_{\eta, \theta} v^{0}\right|_{\eta=0^{+}}+\left.B_{2}(\theta / \varepsilon) \cdot \partial_{\theta} \nabla_{\eta, \theta} v^{0}\right|_{\eta=0^{+}}\right) \\
& -\left.\varepsilon\left(\sigma_{0}-\sigma_{m}\right) n_{\mathcal{C}_{1}} \cdot \nabla_{\eta, \theta} v^{1}\right|_{\eta=0^{+}}+O\left(\varepsilon^{2}\right), \\
{\left[\sigma \partial_{\eta} w^{1}\right]_{\eta=0} } & =-\varepsilon\left(\left.\kappa(\theta) D_{1} \cdot \nabla_{\eta, \theta} v^{0}\right|_{\eta=0^{+}}+\left.D_{2} \cdot \partial_{\theta} \nabla_{\eta, \theta} v^{0}\right|_{\eta=0^{+}}\right) \\
& +\left.\varepsilon\left(\sigma_{0}-\sigma_{m}\right) n_{\mathcal{C}_{0}} \cdot \nabla_{\eta, \theta} v^{1}\right|_{\eta=0^{+}},
\end{aligned}
$$

and

$$
\left.\left[w^{1}\right]\right|_{\gamma_{\varepsilon}}=0,\left.\quad\left[w^{1}\right]\right|_{\eta=0}=0 .
$$

We now introduce the two following problems defined in $\mathbb{R} \times \mathbb{T}$. For $j=1,2$, let $\left(A^{1, j}, a^{1, j}\right)$ with $A^{1, j}$ continuous, satisfy

$$
\begin{aligned}
& \Delta A^{1, j}=G_{j}, \\
& \left.\sigma_{0} \partial_{n} A^{1, j}\right|_{\mathcal{C}_{1}^{+}}-\left.\sigma_{m} \partial_{n} A^{1, j}\right|_{\mathcal{C}_{1}^{-}}=B_{j}, \\
& \left.\sigma_{m} \partial_{n} A^{1, j}\right|_{\mathcal{C}_{0}^{+}}-\left.\sigma_{1} \partial_{n} A^{1, j}\right|_{\mathcal{C}_{0}^{-}}=-D_{j}, \\
& A j^{1} \rightarrow_{x \rightarrow-\infty} 0, A^{1, j} \rightarrow_{x \rightarrow+\infty} a^{1, j} .
\end{aligned}
$$


According to Lemma 2.2 and Remark 2.3 and using (21) it is clear that the above problems are well posed.

We define now the boundary layer corrector of order $1, v_{B L}^{1}$ on $\mathcal{O}$ by:

$$
\begin{aligned}
\forall \eta>0, & \\
v_{B L}^{1}(\eta, \theta) & =\varepsilon^{2}\left(\left.\kappa(\theta)\left[A^{1,1}(\eta / \varepsilon, \theta / \varepsilon)-a^{1,1}\right] \cdot \nabla_{\eta, \theta} v^{0}\right|_{\eta=0^{+}}\right. \\
& +\left.\left[A^{1,2}(\eta / \varepsilon, \theta / \varepsilon)-a^{1,2}\right] \cdot \partial_{\theta} \nabla_{\eta, \theta} v^{0}\right|_{\eta=0^{+}} \\
& \left.+\left.\left[A^{0}(\eta / \varepsilon, \theta / \varepsilon)-a^{0}\right] \cdot \nabla_{\eta, \theta} v^{1}\right|_{\eta=0^{+}}\right), \\
\forall \eta<0, & \\
v_{B L}^{1}(\eta, \theta) & =\varepsilon^{2}\left(\left.\kappa(\theta) A^{1,1}(\eta / \varepsilon, \theta / \varepsilon) \cdot \nabla_{\eta, \theta} v^{0}\right|_{\eta=0^{+}}\right. \\
& \left.+\left.A^{1,2}(\eta / \varepsilon, \theta / \varepsilon) \cdot \partial_{\theta} \nabla_{\eta, \theta} v^{0}\right|_{\eta=0^{+}}+\left.A^{0}(\eta / \varepsilon, \theta / \varepsilon) \cdot \nabla_{\eta, \theta} v^{1}\right|_{\eta=0^{+}}\right) .
\end{aligned}
$$

\section{Justification of the expansion}

Suppose now that $g$ belongs to $H^{7 / 2}(\partial \Omega)$. Then, $v^{0}$ belongs to $H^{4}\left(\mathcal{O}^{0}\right)$ and $H^{4}\left(\mathcal{O}^{1}\right)$. With this regularity, and denoting by $W^{1}=W^{0}-\varepsilon v^{1}-v_{B L}^{1}$, we may differentiate $W^{1}$. Our inductive construction leads to the following problem satisfied by $W^{1}$ :

$$
\begin{aligned}
& \Delta_{\eta, \theta} W^{1}=F_{\varepsilon}(\eta, \theta), \text { in } \mathcal{O}^{1} \cup \mathcal{O}_{\varepsilon}^{m} \cup \mathcal{O}_{\varepsilon}^{0}, \\
& \left.\left(1+d_{0} \kappa\right) \partial_{\eta} W^{1}\right|_{\eta=d_{0}}+\left.\Lambda_{0} W^{1}\right|_{\eta=d_{0}}=g_{1+}^{\varepsilon}, \\
& \left.\left(1-d_{0} \kappa\right) \partial_{\eta} W^{1}\right|_{\eta=-d_{0}}-\left.\Lambda_{1} W^{1}\right|_{\eta=-d_{0}}=g_{1-}^{\varepsilon},
\end{aligned}
$$

with the following transmission conditions:

$$
\begin{aligned}
& \left.\sigma_{0} \partial_{n}^{\Phi} W^{1}\right|_{\gamma_{\varepsilon}^{+}}=\left.\sigma_{m} \partial_{n}^{\Phi} W^{1}\right|_{\gamma_{\varepsilon}^{-}}+\varepsilon^{2} R_{1}^{\varepsilon}, \\
& \left.\sigma_{m} \partial_{\eta} W^{1}\right|_{\eta=0^{+}}=\left.\sigma_{1} \partial_{\eta} W^{1}\right|_{\eta=0^{-}}+\varepsilon^{2} R_{2}^{\varepsilon},
\end{aligned}
$$

and

$$
\begin{aligned}
& \left.W^{1}\right|_{\gamma_{\varepsilon}^{+}}=\left.W^{1}\right|_{\gamma_{\varepsilon}^{-}}, \\
& \left.W^{1}\right|_{\eta=0^{+}}=\left.W^{1}\right|_{\eta=0^{-}}+\varepsilon^{2}\left(\left.\kappa a^{1,1} \cdot \nabla_{\eta, \theta} v^{0}\right|_{\eta=0^{+}}+\left.a^{1,2} \cdot \partial_{\theta} \nabla_{\eta, \theta} v^{0}\right|_{\eta=0^{+}}\right. \\
& \left.+\left.a^{0} \cdot \nabla_{\eta, \theta} v^{1}\right|_{\eta=0^{+}}\right) .
\end{aligned}
$$

The function $F_{\varepsilon}$ is smooth wih respect to the $\eta$-variable while it is squareintegrable in the $\theta$-variable. Moreover it satisfies :

$$
\left|F_{\varepsilon}(\eta, .)\right|_{L^{2}(\mathbb{T})} \leq\left\{\begin{array}{l}
C \varepsilon, \text { if } \eta \leq c \varepsilon \\
\text { decays exponentially for } \eta / \varepsilon \text { tending to infinity. }
\end{array}\right.
$$


which implies that for all $s \in[1,2]$ there exists $c_{s}>0$ such that

$$
\left\|F_{\varepsilon}\right\|_{L^{s}(\mathcal{O})} \leq c_{s} \varepsilon^{1+1 / s}
$$

Simple calculations lead to the following estimates

$$
\begin{aligned}
& \left|R_{1}^{\varepsilon}\right|_{L^{2}\left(\gamma_{\varepsilon}\right)} \leq C, \\
& \left|R_{2}^{\varepsilon}\right|_{L^{2}\left(\gamma^{0}\right)} \leq C, \\
& \left|g_{1 \pm}^{\varepsilon}\right|_{L^{2}\left(\gamma^{ \pm d_{0}}\right)} \leq C_{1} e^{-C_{2} / \varepsilon} .
\end{aligned}
$$

Theorem 1.1 is a straightforward consequence of the following theorem:

Theorem 4.1. For any $s \in] 1,2]$ there exists $c_{s}>0$ independent of $\varepsilon$ such that

$$
\left\|W^{1}\right\|_{H^{1}\left(\mathcal{D}^{1}\right)}+\left\|W^{1}\right\|_{H^{1}\left(\mathcal{D}^{0}\right)} \leq c_{s} \varepsilon^{1+1 / s} .
$$

Proof. To prove the estimate, we write the problem satisfied by $W^{1}$ in local $(\eta, \theta)$-coordinates. We consider a function $p \in H^{2}\left(\mathcal{O}^{1}\right)$ such that

$$
\begin{aligned}
& p=\left.\kappa a^{1,1} \cdot \nabla_{\eta, \theta} v^{0}\right|_{\eta=0^{+}}+\left.a^{1,2} \cdot \partial_{\theta} \nabla_{\eta, \theta} v^{0}\right|_{\eta=0^{+}}+\left.a^{0} \cdot \nabla_{\eta, \theta} v^{1}\right|_{\eta=0^{+}} \quad \text { on } \eta=0, \\
& \partial_{\eta} p=0 \quad \text { on } \eta=0 \\
& p=\partial_{\eta} p=0 \quad \text { on } \eta=-d_{0}
\end{aligned}
$$

and we set

$$
\hat{W}^{1}=W^{1}+\varepsilon^{2} p 1_{\mathcal{O}^{1}},
$$

where $1_{\mathcal{O}^{1}}$ denotes the characteristic function of $\mathcal{O}^{1}$. Then $\hat{W}^{1}$ is continuous and satisfies:

$$
\begin{aligned}
& \Delta_{\eta, \theta} \hat{W}^{1}=F_{\varepsilon}(\eta, \theta), \text { in } \mathcal{O}_{\varepsilon}^{m} \cup \mathcal{O}_{\varepsilon}^{0}, \\
& \Delta_{\eta, \theta} \hat{W}^{1}=F_{\varepsilon}(\eta, \theta)+\varepsilon^{2} \Delta_{\eta, \theta} p, \text { in } \mathcal{O}^{1}, \\
& \left.\left(1+d_{0} \kappa\right) \partial_{\eta} \hat{W}^{1}\right|_{\eta=d_{0}}+\left.\Lambda_{0} \hat{W}^{1}\right|_{\eta=d_{0}}=g_{1+}^{\varepsilon}, \\
& \left.\left(1-d_{0} \kappa\right) \partial_{\eta} \hat{W}^{1}\right|_{\eta=-d_{0}}-\left.\Lambda_{1} \hat{W}^{1}\right|_{\eta=-d_{0}}=g_{1-}^{\varepsilon}, \\
& \left.\sigma_{0} \partial_{n}^{\Phi} \hat{W}^{1}\right|_{\gamma_{\varepsilon}^{+}}=\left.\sigma_{m} \partial_{n}^{\Phi} \hat{W}^{1}\right|_{\gamma_{\varepsilon}^{-}}+\varepsilon^{2} R_{1}^{\varepsilon}, \\
& \left.\sigma_{m} \partial_{\eta} \hat{W}^{0}\right|_{\eta=0^{+}}=\left.\sigma_{1} \partial_{\eta} \hat{W}^{0}\right|_{\eta=0^{-}}+\varepsilon^{2} R_{2}^{\varepsilon} .
\end{aligned}
$$

Now multiplying the main equations by $\sigma(1+\eta \kappa) \varphi$ and integrating by parts, we infer the following variational formulation for $\hat{W}^{1} \in H^{1}(\mathcal{O})$ :

$$
\begin{aligned}
& \int_{\mathcal{O}} \sigma D \nabla_{\eta, \theta} \hat{W}^{1} \cdot \nabla_{\eta, \theta} \varphi+\sigma_{0}<\Lambda_{0} \hat{W}^{1}, \varphi>+\sigma_{1}<\Lambda_{1} \hat{W}^{1}, \varphi>=-\int_{\mathcal{O}} \sigma(1+\eta \kappa) F_{\varepsilon} \varphi \\
& -\varepsilon^{2} \sigma^{1} \int_{\mathcal{O}}(1+\eta \kappa) \Delta_{\eta, \theta} p \varphi+\sigma^{0} \int_{\gamma^{d_{0}}} g_{1+}^{\varepsilon} \varphi-\sigma^{1} \int_{\gamma^{-d_{0}}} g_{1-\varphi}^{\varepsilon}, \\
& -\varepsilon^{2} \int_{\gamma_{\varepsilon}} \frac{\sqrt{(1+\varepsilon \kappa f)^{2}+\left(f^{\prime}\right)^{2}}}{\sqrt{1+\left(f^{\prime}\right)^{2}}} R_{1}^{\varepsilon} \varphi-\varepsilon^{2} \int_{\gamma^{0}} R_{2}^{\varepsilon} \varphi, \quad \forall \varphi \in H^{1}(\mathcal{O}),
\end{aligned}
$$

where $D$ is the diagonal matrix with elements $1+\eta \kappa$ and $\frac{1}{1+\eta \kappa}$.

Taking $\varphi=\hat{W}^{1}$, using the inequalities (22) and (23) and using also a result of Bonder et al. [5], since the amplitude and the period of the oscillations of $\Gamma_{\varepsilon}^{r}$ have the same order, we infer the result. 
Remark 4.2. Since the boundary layer corrector $v_{B L}^{1}$ is of order $\varepsilon^{2}$ in $L^{2}(\mathcal{O})$ we deduce for any $s \in] 1,2]$ :

$$
\left\|v^{\varepsilon}-v^{0}-v_{B L}^{0}-\varepsilon v^{1}\right\|_{L^{2}(\mathcal{O})} \leq C_{s} \varepsilon^{1+1 / s}
$$

\section{Acknowledgements}

The authors thank very warmly $\mathrm{H}$. Ammari for his well-considered advice and suggestions.

\section{References}

[1] T. Abboud and H. Ammari. Diffraction at a curved grating: TM and TE cases, homogenization. J. Math. Anal. Appl., 202(3):995-1026, 1996.

[2] Y. Achdou and O. Pironneau. Domain decomposition and wall laws. C. R. Acad. Sci. Paris Sér. I Math., 320(5):541-547, 1995.

[3] Grégoire Allaire and Micol Amar. Boundary layer tails in periodic homogenization. ESAIM Control Optim. Calc. Var., 4:209-243 (electronic), 1999.

[4] A. Basson and D. Gérard-Varet. Wall laws for fluid flows at a boundary with random roughness. To appear in Comm. Pure Applied Math., 2008.

[5] Julián Fernández Bonder, Rafael Orive, and Julio D. Rossi. The best Sobolev trace constant in a domain with oscillating boundary. Nonlinear Anal., 67(4):1173-1180, 2007.

[6] Willi Jäger, Andro Mikelić, and Nicolas Neuss. Asymptotic analysis of the laminar viscous flow over a porous bed. SIAM J. Sci. Comput., 22(6):20062028 (electronic), 2000.

[7] C. Poignard. Approximate transmission conditions through a weakly oscillating thin layer. Math. Meth. App. Sci., 2008. To appear. 18p. http://www.cmap.polytechnique.fr/ poignard/membWosci-18pp.html.

[8] C. Poignard, P. Dular, R. Perrussel, L. Krähenbühl, L. Nicolas, and M. Schatzman. Approximate condition replacing thin layer. IEEE Trans. on Mag., 44(6):1154-1157, 2008. http://hal.archives-ouvertes.fr/hal$00165049 /$ fr.

[9] T. Saarenketo. Electrical properties of water in clay and silty soils. Journal of Applied Geophysics, 40:73-88, 1998.

[10] D. Wildenschild, J.J. Roberts, and E.D. Carlberg. Electrical properties of sand-clay mixtures: the effect of microstructure. Technical report, July 1999. 
Centre de recherche INRIA Bordeaux - Sud Ouest

Domaine Universitaire - 351, cours de la Libération - 33405 Talence Cedex (France)

Centre de recherche INRIA Grenoble - Rhône-Alpes : 655, avenue de l'Europe - 38334 Montbonnot Saint-Ismier

Centre de recherche INRIA Lille - Nord Europe : Parc Scientifique de la Haute Borne - 40, avenue Halley - 59650 Villeneuve d'Ascq

Centre de recherche INRIA Nancy - Grand Est : LORIA, Technopôle de Nancy-Brabois - Campus scientifique 615, rue du Jardin Botanique - BP 101 - 54602 Villers-lès-Nancy Cedex

Centre de recherche INRIA Paris - Rocquencourt : Domaine de Voluceau - Rocquencourt - BP 105 - 78153 Le Chesnay Cedex

Centre de recherche INRIA Rennes - Bretagne Atlantique : IRISA, Campus universitaire de Beaulieu - 35042 Rennes Cedex

Centre de recherche INRIA Saclay - Île-de-France : Parc Orsay Université - ZAC des Vignes : 4, rue Jacques Monod - 91893 Orsay Cedex

Centre de recherche INRIA Sophia Antipolis - Méditerranée : 2004, route des Lucioles - BP 93 - 06902 Sophia Antipolis Cedex 